\title{
A rare case of chromophobe renal cell carcinoma accompanied by abdominal compartment syndrome
}

\author{
Kimihiko Masui • Yuka Shimane $\cdot$ Yuzuru Megumi • \\ Hideki Nosaka $\cdot$ Mitsuhiro Tachibana $\cdot$ \\ Shigeki Fukuzawa
}

Received: 29 August 2012/Accepted: 29 October 2012/Published online: 5 December 2012

(C) The Japan Society of Clinical Oncology 2012

\begin{abstract}
We report a rare case of chromophobe renal cell carcinoma with abdominal compartment syndrome (ACS). A 45-year-old man was admitted to our hospital with severe right back pain. Abdominal computed tomography revealed a $20 \mathrm{~cm}$ bleeding tumor in the right kidney. We performed transarterial embolization (TAE) of the right renal artery and capsular branches. After TAE, the condition of the patient deteriorated, resulting in multiple organ failure. On day 6 after admission, open right nephrectomy was performed through a transperitoneal approach. He soon recovered from multiple organ failure and was discharged from hospital 31 days postoperatively. We confirmed that the preoperative organ failures caused intra-abdominal hypertension, and thus attributed the lifethreatening symptoms to ACS. This represents the first report of renal cell carcinoma presenting as ACS.
\end{abstract}

Keywords Abdominal compartment syndrome (ACS) . Intra-abdominal hypertension (IAH) - Round belly sign (RBS) - Multiple organ failure $\cdot$ Renal cell carcinoma (RCC)

K. Masui $(\bowtie) \cdot$ Y. Shimane · Y. Megumi - S. Fukuzawa Department of Urology, Shimada Municipal Hospital, 1200-5 Noda, Shimada, Shizuoka 427-8502, Japan e-mail: kmasui@municipal-hospital.shimada.shizuoka.jp

H. Nosaka

Department of Nephrology, Shimada Municipal Hospital, Shimada, Shizuoka, Japan

M. Tachibana

Department of Diagnostic Pathology, Shimada Municipal Hospital, Shimada, Shizuoka, Japan

\section{Introduction}

Chromophobe renal cell carcinoma (ChRCC), first described by Thoenes et al. in 1985, represents 3-5\% of all RCCs. Prognosis for ChRCC appears to be better than the prognoses for other types of RCC [1].

Abdominal compartment syndrome (ACS) represents the natural progression of end-organ dysfunction, and develops if intra-abdominal hypertension (IAH) is not recognized appropriately.

We report a rare case of ChRCC that presented as ACS and was treated by surgical resection.

\section{Case report}

A 45-year-old man was brought to our hospital by ambulance with complaints of right back pain continuing from the previous day. His medical history included hypertension, hyperlipidemia, hyperuricemia, and obesity, and he was receiving treatment for all of these at a local clinic.

In our emergency department, the patient showed both hypertension and a distended abdomen during initial examination, but complained of no other symptoms apart from his back pain. Results of laboratory evaluations were as follows: white blood cell count (WBC), $11.1 \times 10^{3} / \mu \mathrm{L}$; hemoglobin level, $11.6 \mathrm{~g} / \mathrm{dL}$; platelet count, $16.5 \times 10^{3} / \mu \mathrm{L}$; alanine aminotransferase (ALT), $38 \mathrm{U} / \mathrm{L}$. Elevated levels of aspartate aminotransferase (AST) $(564 \mathrm{U} / \mathrm{L})$ and lactate dehydrogenase (LDH) ( $3280 \mathrm{U} / \mathrm{L})$ were also noted. However, levels of creatinine (CRE) were not elevated at that time $(0.64 \mathrm{mg} / \mathrm{dl})$. In the arterial phase of computed tomography (CT), a bleeding tumor $20 \mathrm{~cm}$ in diameter, was revealed in the right kidney. The inferior vena cava (IVC) was severely 


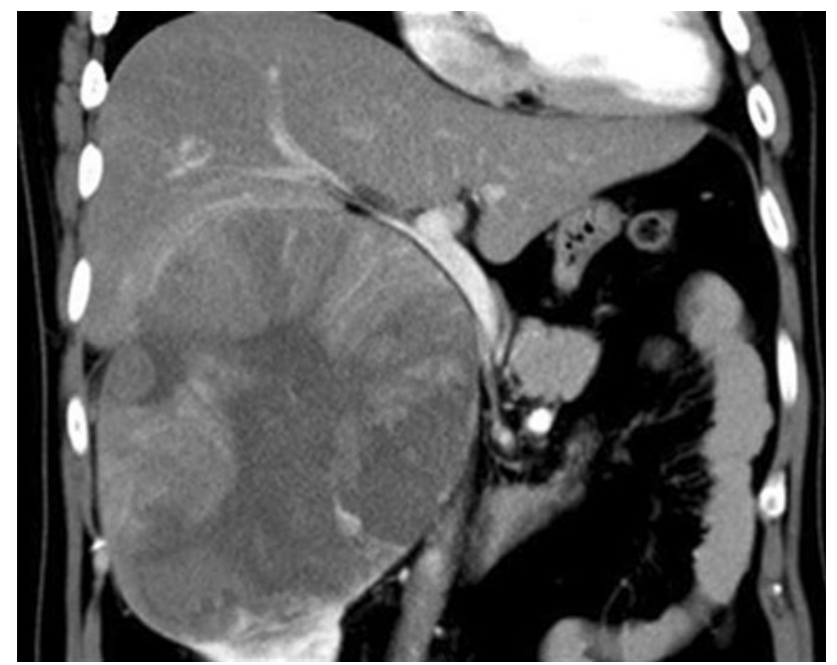

Fig. 1 Computed tomography (CT) of the abdomen showing enhanced bleeding tumor, $20 \mathrm{~cm}$ in diameter, in the right kidney during the arterial phase; the inferior vena cava (IVC) was severely compressed by the tumor

compressed by the right renal tumor (Fig. 1), and the patient was promptly admitted to our hospital.

On day 2, he showed tachycardia, tachypnea, and deteriorating anemia (hemoglobin, $9.7 \mathrm{~g} / \mathrm{dL}$ ). We therefore performed transarterial embolization (TAE) of the right renal artery and capsular branches. However, his condition deteriorated, resulting in multiple organ failure.

On day 3 , his vital signs were as follows: blood pressure, $170 / 110 \mathrm{mmHg}$; heart rate, 110 beats/min; and respiratory rate, 40 breaths/min. Pulse oximetry saturation $\left(\mathrm{SpO}_{2}\right)$ was $90 \%$ (oxygen, $5 \mathrm{~L} / \mathrm{min}$ delivered by mask). Physical examination showed that the abdomen had further distended since the day of admission, and discoloration appeared on the legs. Ultrasonography revealed both decreasing pulmonary circulation and a decreasing ejection fraction. Laboratory evaluations were as follows: WBC, $16.7 \times 10^{3} / \mu \mathrm{L}$; hemoglobin level, $7.7 \mathrm{~g} / \mathrm{dL}$; platelet count, $14.3 \times 10^{3} / \mu \mathrm{L} ;$ AST, $2329 \mathrm{U} / \mathrm{L} ;$ ALT, $151 \mathrm{U} / \mathrm{L} ; \mathrm{LDH}$, $29184 \mathrm{U} / \mathrm{L}$; and CRE, $2.45 \mathrm{mg} / \mathrm{dl}$. Arterial blood gas analysis showed that the partial pressure of oxygen $\left(\mathrm{PaO}_{2}\right)$ was $65 \mathrm{mmHg}$ and that of carbon dioxide $\left(\mathrm{PaCO}_{2}\right)$ was $42 \mathrm{mmHg}$ (oxygen, $5 \mathrm{~L} / \mathrm{min}$ delivered by mask). Chest radiography revealed the diaphragm was displaced cephalad (Fig. 2a). We therefore started continuous hemodiafiltration, noninvasive positive-pressure ventilation, and additional interventions.

Laboratory evaluations were unimproved on day 4 , particularly CRE $(3.79 \mathrm{mg} / \mathrm{dl})$. The patient complained of nausea and his abdomen was paralytic on examination. Abdominal radiography revealed a distended large intestine (Fig. 3). Because of the rapid exacerbation, we considered
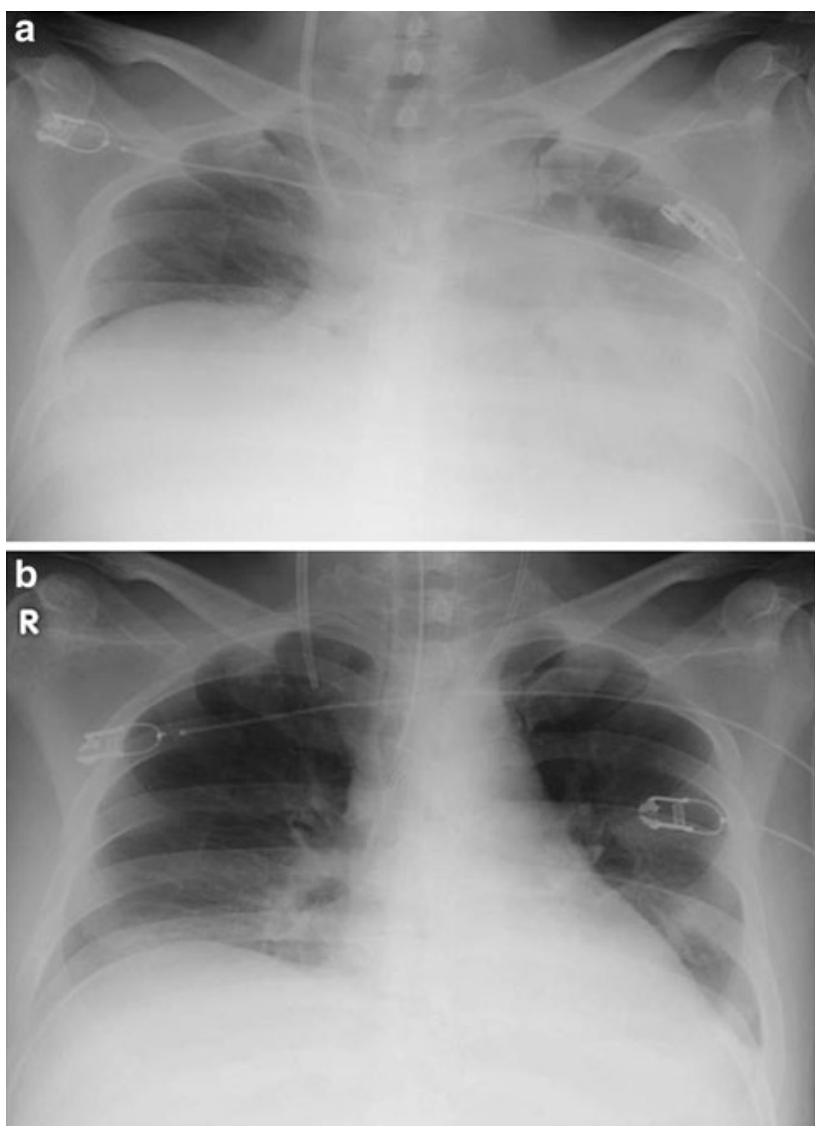

Fig. 2 Chest X-rays, sitting position. a The diaphragm was displaced cephalad on the day before surgery; $\mathbf{b}$ when checked on postoperative day 1 , the position of the diaphragm had normalized

the symptoms to be closely associated with ACS. We therefore measured intra-abdominal pressure (IAP) and confirmed IAH (24 mmHg), and, based on these results, decided that surgery was necessary in this situation.

Open right nephrectomy was performed through a transperitoneal approach. An accumulation of about $100 \mathrm{~mL}$ of blood in the abdomen as well as a distended bowel were observed on incision. The tumor was removed without incident even though it had adhered to the ascending colon, IVC, duodenum, and pancreas.

The resected tumor measured $19 \times 18 \times 13 \mathrm{~cm}$ and weighed $3500 \mathrm{~g}$. The histopathological diagnosis was a ChRCC, eosinophilic variant, classification pT2b, with negative surgical margins. Under the microscope, the tumor mostly comprised coagulative necrosis of tumor tissue (Fig. 4). Immunohistochemically, positive results were seen for c-kit and CD10.

He soon recovered from multiple organ failure, the position of his diaphragm normalized (Fig. 2b), and his IAP decreased to $<10 \mathrm{mmHg}$. He was discharged from our hospital 31 days postoperatively. 


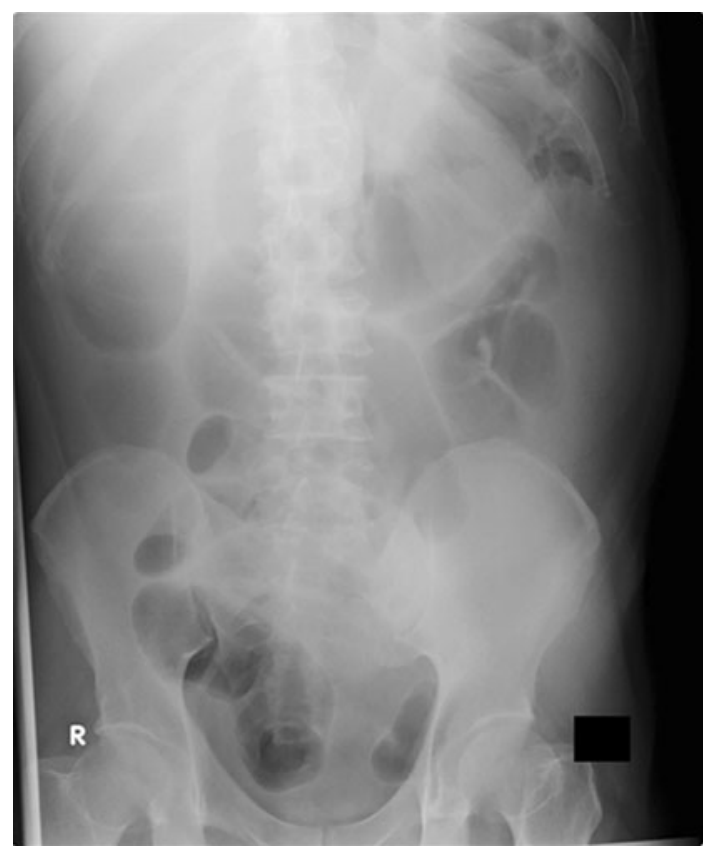

Fig. 3 Abdominal X-rays, recumbent position. On day 4 after admission, abdominal radiography revealed a distended large intestine

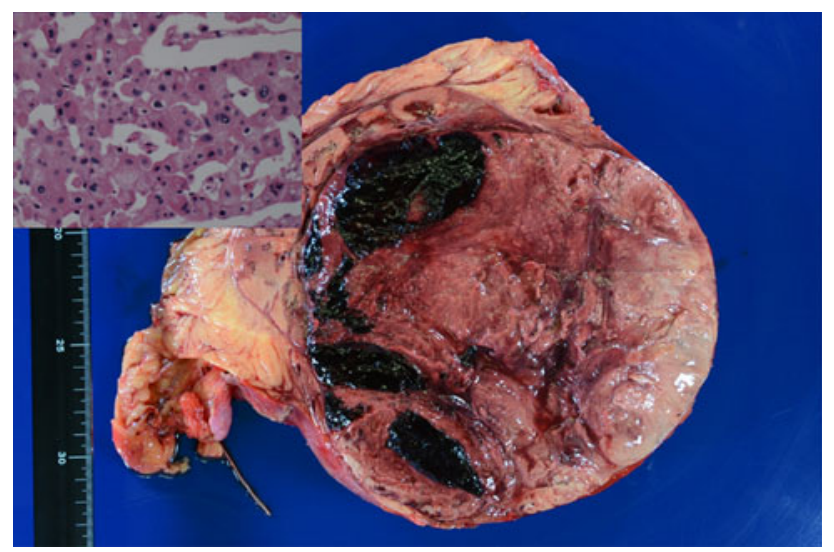

Fig. 4 The tumor, which comprised solid cell sheets with massive cell necrosis. Tumor nuclei were round to oval in shape, larger than red blood cells in size, and contained coarse chromatin and small nucleoli. The nucleocytoplasmic ratio is generally low. The cytoplasm shows characteristic eosinophilic granules. Immunohistologically, the tumor cells show positive staining for c-kit (CD117) and CD10

\section{Discussion}

Historically, IAH and ACS have not been prominent concepts. However, they have been gaining increasing recognition as critical factors in treatment of the severely ill over the past decade [2].

It was not until 2006 that the World Society on Abdominal Compartment Syndrome (WSACS) established consensus definitions for IAH and ACS. IAH is defined as a sustained or repeated pathological elevation in IAP to $\geq 12 \mathrm{mmHg}$. ACS is defined as a sustained IAP $>20 \mathrm{mmHg}$ (with or without abdominal perfusion pressure $<60 \mathrm{mmHg}$ ) that is associated with new organ dysfunction/failure [3]. Due to its simplicity and low cost, intravesical IAP measurement has been considered the gold standard [2]. Radiologically, Al-Bahrani et al. [4] reported that the presence of round belly sign (RBS) and bowel wall thickening with enhancement on CT in critically ill surgical patients should alert clinicians to the possible presence of IAH and ACS. Pickhardt et al. [5] defined RBS as an increased ratio (ratio $>0.80$ ) of anteroposterior:transverse diameters measured at the level where the left renal vein crosses the aorta and excluding subcutaneous fat.

The presentation of the present case was unusual for RCC. We therefore report and discuss the mechanisms involved in renal tumor and ACS.

We confirmed multiple organ failure involving the renal, respiratory, gastrointestinal, and hepatic systems. Increased IAP not only decreases regional blood flow in the peritoneal cavity, but also exerts adverse effects on organs and systems outside the abdomen. In particular, direct vascular and organ compression and diaphragmatic elevation are the key mechanisms leading to the circulatory collapse that lies at the center of the pathophysiology of ACS [6]. We thus consider the multiple organ failure in this case to be closely associated with ACS.

In this case, the tumor compressed IVC because of its large size, leading to decreased ventricular pre-load and increased afterload. These processes decreased cardiac output and reduced blood flow to multiple organs. In addition, the same mechanisms resulted in IAP and diaphragmatic elevation, which then led to reductions in static and dynamic pulmonary compliance [6]. These multifactorial processes caused multiple organ failure involving the renal, respiratory, gastrointestinal, and hepatic systems. Actually, Saggi et al. [6] reviewed previous reports about ACS and the threshold IAP for organ failure, finding it was likely to be caused by IAP $>20 \mathrm{mmHg}$. In the present case, preoperative IAP was $24 \mathrm{mmHg}$.

The mechanisms that led to IAH in this case are unclear. We suppose that sudden hemorrhage of the growing renal tumor would have resulted in severe pain. As the patient endured this severe pain for one day, distended abdomen and IAH developed. His obesity would have exacerbated the situation. From the initial CT taken in our emergency department, we were able to confirm RBS.

The renal tumor was diagnosed as ChRCC. Some authors have reported that poor prognostic factors for ChRCC include clinical/pathological $\mathrm{T}$ stage, tumor size $>7 \mathrm{~cm}$, small-vessel invasion, sarcomatoid differentiation, microscopic necrosis, and CD10-positive status [1, 7, 8]. This case involved a large tumor composed of solid cell 
sheets with massive cell necrosis, and immunohistochemical examination showed CD10-positive results. These features all indicate an aggressive tumor.

This represents the first report of renal cell carcinoma presenting as ACS. When treating a large retroperitoneal tumor, the possibility of ACS should be considered.

Conflict of interest The authors declare that they have no conflict of interest.

\section{References}

1. Volpe A, Novara G, Antonelli A et al (2011) Chromophobe renal cell carcinoma: oncological outcomes and prognostic factors in a large multicentre series. BJU Int 110:76-83

2. Cheatham ML, Malbrain ML, Kirkpatrick A et al (2007) Results from the International Conference of Experts on Intra-abdominal Hypertension and Abdominal Compartment Syndrome. II. Recommendations. Intensive Care Med 33:951-962
3. Malbrain ML, Cheatham ML, Kirkpatrick A et al (2006) Results from the International Conference of Experts on Intra-abdominal Hypertension and Abdominal Compartment Syndrome. I. Definitions. Intensive Care Med 32:1722-1732

4. Al-Bahrani AZ, Abid GH, Sahgal E et al (2006) A prospective evaluation of CT features predictive of intra-abdominal hypertension and abdominal compartment syndrome in critically ill surgical patients. Clin Radiol 62:676-682

5. Pickhardt PJ, Shimony JS, Heiken JP et al (1999) The abdominal compartment syndrome: CT findings. Am J Roentgenol 173:575-579

6. Saggi BH, Sugerman HJ, Ivatury RR et al (1998) Abdominal compartment syndrome. J Trauma 45:597-609

7. Przybycin CG, Cronin AM, Darvishian F et al (2011) Chromophobe renal cell carcinoma: a clinicopathologic study of 203 tumors in 200 patients with primary resection at a single institution. Am J Surg Pathol 35:962-970

8. Martignoni G, Pea M, Brunelli M et al (2004) CD10 is expressed in a subset of chromophobe renal cell carcinomas. Mod Pathol 17:1455-1463 